\title{
Elastic properties of the ascending aorta in patients with rheumatoid arthritis
}

\author{
Enrico Vizzardi a,*, Ilaria Cavazzana ${ }^{\text {b }}$, Natalia Pezzali a , Angela Ceribelli ${ }^{\text {b }}$, Chiara Bazzani ${ }^{\text {b }}$, Angela Tincani ${ }^{\text {b }}$, \\ Marco Metra ${ }^{a}$, Franco Franceschini ${ }^{b}$, Livio Dei Cas ${ }^{a}$ \\ a Cardiovascular Disease Section, Department of Applied Experimental Medicine, University of Brescia, Italy \\ ${ }^{\mathrm{b}}$ Rheumatology Unit and Chair, University of Brescia, Italy
}

A R T I C L E I N F O

Article history:

Received 30 April 2011

Accepted 14 May 2011

Keywords:

Rheumatoid arthritis

Aortic stiffness and distensibility

Left ventricular mass mass

Left ventricular diastolic function

Systemic inflammatory rheumatic diseases, such as rheumatoid arthritis (RA), are associated with increased cardiovascular mortality, on account of the high prevalence of ischemic heart disease and accelerated atherosclerosis, compared with age- and sex-matched controls [1]. The higher risk of cardiovascular disease (CVD) in RA patients seems to be independent of traditional cardiovascular risk factors. Different pathogenic mechanisms including pro-oxidative dyslipidemia, insulin resistance, prothrombotic state and hyperhomocysteinemia have been described [2]. However, immune activation is a key mechanism that promotes structural and functional abnormalities of the vascular bed. Arterial stiffness, usually measured by pulse-wave velocity analysis, is considered a marker of subclinical vascular disease and increased CVD risk, and it is markedly abnormal in patients with RA [3,4]. Large left ventricular (LV) mass has also been associated with RA, suggesting a link between chronic inflammation and LV hypertrophy [5]. We investigated the elastic properties of the aorta and systo-diastolic function in RA patients without CV disease, compared with healthy controls.

In our study, RA was diagnosed according to American College of Rheumatology criteria [6]. Disease activity was assessed at time of cardiological evaluation using a composite index, called DAS44, including swollen and tender joint count (on 44 joints), global health assessment and ESR value. The patients had a cardiological examination, 12-lead electrocardiography (ECG) and two-dimensional and Doppler transthoracic echocardiography. The exclusion criteria of the study were: (a) arterial hypertension (blood pressure $>140 / 90 \mathrm{mmHg}$ in more than three consecutive readings or use of any hypotensive drugs); (b) diabetes; (c) smoke; (d) symptomatic dyspnea or chest pain; (e) use of any cardiovascular drugs (including statins); (g) any previous myocardial infarction, surgical or percutaneous revascularization, a positive ECG result, perfusion or echocardiographic exercise or pharmacological stress test; (h) more than mild aortic or mitral regurgitation and/or stenosis; (i) any previous surgical or interventional cardiac or vascular procedure; (l) familial hypercholesterolemia; (m) any genetic cardiovascular disease (including cardiomyopathy or Marfan syndrome). Patients were matched to normal controls for age, sex and ethnicity.

The 44 selected RA patients have a mean age of 55 years ( \pm 14 years) and mean duration of RA 9.6 years ( \pm 7.6 years). CRP was high at the time of the cardiac evaluation in 19 patients (43.2\%). Rheumatoid factor and anti-cyclic citrullinated peptide (anti-CCP) antibodies were found in $73.8 \%$ and $62 \%$ of subjects, respectively. The mean disease activity index, assessed using the DAS44 score, was low (mean $2.4 \pm 1.6$ ). Prednisone,

\footnotetext{
* Corresponding author at: Pzzle Spedali civili 1, 25100 Brescia, Italy. Tel.: + 39030 3995659; fax: + 390303995061.

E-mail address: enrico.vizzardi@tin.it (E. Vizzardi).
}

methotrexate, or anti-TNF agents were currently being or had been used by respectively $95 \%, 80.5 \%$ and $63.4 \%$ of the patients. Table 1 shows their baseline demographic and cardiovascular data.

Compared with age- and sex-matched controls, the RA patients had greater posterior wall and septal thickness, but no other differences from controls in terms of LV end-diastolic/end-systolic diameter or ejection fraction (Table 2). As a consequence, RA subjects had a greater LV mass ( $155 \pm 47$ vs. $140 \pm 31 \mathrm{~g} ; p=0.01)$, LV mass index (mean $140 \pm 31$ vs. $92 \pm 25 \mathrm{~g} / \mathrm{m}^{2} p<0.001$ ) and indexing to height in meters to the power 2.7 ( $43 \pm 12$ vs. $31 \pm 10 \mathrm{~g} / \mathrm{m}^{2.7}$; $p=0.0043$ ) than controls.

We also found lower mean aortic strain $(7.7 \pm 3 \%$ vs. $13 \pm 5 \%$; $p<0.001)$ and distensibility $\left(2.8 \pm 1.2\right.$ vs. $5.4 \pm 2.6 \mathrm{~cm}^{2} \mathrm{dyn}^{-1} \times 10^{-6}$; $p<0.001)$ with a higher mean stiffness index $(9.2 \pm 6.05$ vs. $5.06 \pm 2.9$; $p<0.001)$ in RA cases, compared with controls. There was also a significant difference between RA patients and controls in term of E/A ( $1.1 \pm 0.7$ vs. $1.7 \pm 0.6, p<0.001)$, deceleration time ( $225 \pm 56$ vs. $190 \pm 50 \mathrm{~ms}, p=0.004)$ and $\mathrm{E} / \mathrm{E}^{\prime}(10.3 \pm 5.5$ vs. $7.3 \pm 3.5, p<0.01)$.

A comparison of aortic distensibility and other morphological and functional cardiac parameters showed no significant association between LV mass with aortic distensibility, aortic strain and stiffness. In addition, RA duration, disease activity index (DAS 44) and CRP did not correlate with either LV mass or aortic elastic properties.

Increased aortic stiffness and decreased aortic distensibility were closely associated with diastolic filling indexes measured by conventional and tissue Doppler echocardiography. In particular, we found a significant association between aortic distensibility, E/A $(p<0.001)$ and $\mathrm{E}^{\prime}(p=0.013)$, aortic stiffness and deceleration time $(p: 0.01)$ and between aortic strain, $\mathrm{E} / \mathrm{A}(p<0.001)$ and $\mathrm{E}^{\prime}(p=0.02)$ After adjustment for age and systolic blood pressure, at multivariable stepwise regression analysis, RA $(p<0.0001)$ and systolic blood pressure $(p=0.022)$ were independently related with distensibility, only RA was independently related with stiffness $(p=0.0003)$ and with strain $(p<0.0001)$. Age $(p=0.0004)$ and RA $(p=0.0014)$ were independently related with $\mathrm{E} / \mathrm{A}$, while only RA $(p=0.0069)$ was independently related with $\mathrm{E} / \mathrm{E}^{\prime}$. RA $(p=0.00154)$ and age $(p=0.0076)$ were independently related with DT.

At a multivariable stepwise regression analysis, rheumatoid factor, DAS, anti-CCP status were not related with aortic stiffness, distensibility, LV mass. Stratifying cases on the basis of RA duration (more or less than ten years), we also found no differences for LV mass and other elastic properties of the aortic wall. When considering RA treatment, prednisone, methotrexate or anti-TNF alpha therapies were not related with stiffness, distensibility, LV mass at multivariable regression analysis.

The results of this study are that, first, patients with RA without CV disease or any CV risk factors had an increase in LV mass and abnormal elastic properties of the aorta (increased arterial stiffness, lower

Table 1

Main-features of the rheumatoid arthritis patients and controls.

\begin{tabular}{llll}
\hline & RA patients $(n .44)$ & Controls $(n .35)$ & $p$ \\
\hline Age (mean \pm SD) & $57 \pm 15$ & $56 \pm 10$ & $\mathrm{~ns}$ \\
Sex $(\%$ female) & $73 \%$ & $71 \%$ & $\mathrm{~ns}$ \\
Systolic pressure mmHg (mean $\pm \mathrm{SD})$ & $130 \pm 11$ & $128 \pm 11$ & $\mathrm{~ns}$ \\
Diastolic pressure mmHg $($ mean $\pm \mathrm{SD})$ & $74 \pm 8$ & $71 \pm 17$ & $\mathrm{~ns}$ \\
Heart rate, beats $/$ minute $($ mean $\pm \mathrm{SD})$ & $76 \pm 10$ & $72 \pm 11$ & $\mathrm{~ns}$ \\
Body surface area (mean $\pm \mathrm{SD})$ & $1.7 \pm 0.2$ & $1.7 \pm 0.4$ & $\mathrm{~ns}$
\end{tabular}


Table 2

Left ventricular (LV) structure and function parameters in RA patients and controls.

\begin{tabular}{lccc}
\hline & RA patients (n. 44) & Controls $(n .35)$ & $p$ \\
\hline Septal thickness mm & $10 \pm 1.2$ & $9.3 \pm 1.2$ & 0.02 \\
Posterior wall thickness mm & $8.9 \pm 1.2$ & $8.2 \pm 1.2$ & 0.01 \\
LV end-diastolic dimension mm & $47 \pm 7$ & $47 \pm 4$ & 0.93 \\
LV end-systolic dimension mm & $25 \pm 3.4$ & $27 \pm 7$ & 0.25 \\
Ejection fraction \% & $62 \pm 7$ & $64 \pm 4.4$ & 0.26 \\
LV mass, g & $155 \pm 47$ & $140 \pm 31$ & 0.012 \\
LV mass index, g/m ${ }^{2}$ & $140 \pm 31$ & $92 \pm 25$ & $<0.0001$ \\
LV mass index, g/m (2.7 $_{\text {E/A }}$ & $43 \pm 12$ & $31 \pm 10$ & 0.0043 \\
DT msec & $1.1 \pm 0.7$ & $1.7 \pm 0.6$ & 0.0003 \\
E/E' & $234 \pm 55$ & $190 \pm 50$ & 0.005 \\
AoS mm & $11 \pm 5.8$ & $7.3 \pm 3.6$ & 0.0034 \\
AoD mm & $3.2 \pm 0.4$ & $3.05 \pm 0.4$ & 0.01 \\
Distensibility cm ${ }^{2}$ dyn $^{-1} 10^{-6}$ & $3 \pm 0.4$ & $2.7 \pm 0.4$ & 0.0054 \\
Stiffness index & $3 \pm 1.2$ & $5.4 \pm 2.7$ & $<0.001$ \\
Strain \% & $8.3 \pm 4.5$ & $5 \pm 2.9$ & 0.002 \\
\hline
\end{tabular}

distensibility and strain) than controls. Second, these patients also had significant impairment of LV diastolic function and a close correlation between this dysfunction and increased aorta stiffness.

The increased LV mass and stiffening of the aorta, even in patients without hypertension or clinical CV disease, suggest that RA patients might have a condition of preclinical atherosclerosis. Aortic elastic properties are important determinants of LV systolic and diastolic function and, in some cases, increased stiffness has been linked to diastolic heart failure. Increased diastolic dysfunction in RA seems to correlate with long disease duration. We found a correlation between aortic stiffness, distensibility and diastolic ventricular dysfunction.
These two conditions probably influence the prognosis of RA patients and provide a plausible mechanism for their increased cardiovascular morbidity and mortality. Although our patients did not have active disease at the time of cardiologic assessment, the longstanding chronic systemic inflammatory state may accelerate the atherosclerotic process, increasing aortic stiffening and LV mass.

Further prospective studies are needed to establish whether abnormal elastic aortic properties really correspond to an increased risk of cardiovascular disease in RA.

The authors of this manuscript have certified that they comply with the Principles of Ethical Publishing in the International Journal of Cardiology [7].

\section{References}

[1] Solomon DH, Karlson EW, Rimm EB, et al. Cardiovascular morbidity and mortality in women diagnosed with rheumatoid arthritis. Circulation 2003;107:1303-7.

[2] Tanasescu C, Jurkut C, Jurcut R, Ginghina C. Vascular disease in rheumatoid arthritis: from subclinical lesions to cardiovascular risk. Eur J Intern Med 2009;20:348-54.

[3] Maki-Petaja KM, Hall FC, Booth AD, et al. Rheumatoid arthritis is associated with increased aortic pulse-wave velocity, which is reduced by antitumor necrosis factor alpha therapy. Circulation 2006;114:1185-92.

[4] Wong M, Toh L, Wilson A, et al. Reduced arterial elasticity in rheumatoid arthritis and the relationship to vascular disease risk factors and inflammation. Arthritis Rheum 2003;48:81-9.

[5] Rudominer RL, Roman MJ, Devereux RB, et al. Independent association of rheumatoid arthritis with increased left ventricular mass but not with reduced ejection fraction. Arthritis Rheum Jan 2009;60(1):22-9.

[6] Arnett FC, Edworthy SM, Bloch DA, et al. The American Rheumatism Association 1987 revised criteria for the classification of rheumatoid arthritis. Arthritis Rheum 1988;31:315-24.

[7] Shewan LG, Coats AJ. Ethics in the authorship and publishing of scientific articles. Int J Cardiol 2010;144:1-2.

\title{
Antigen carbohydrate 125 in heart failure: A promising clinical tool
}

\author{
Julio Núñez *, Gema Miñana, Eduardo Núñez, Juan Sanchis \\ Servicio de Cardiología, Hospital Clínic Universitari, INCLIVA, Universitat de Valencia, Valencia-Spain
}

\section{A R T I C L E I N F O}

\section{Article history:}

Received 29 April 2011

Accepted 14 May 2011

Available online 2 June 2011

\section{Keywords:}

Antigen carbohydrate 125

Serosal effusion

Heart failure

Prognosis

\section{Dear editor,}

We want to acknowledge Topatan and Başaran for their contribution toward a better understanding of the pathophysiology of antigen carbohydrate 125 (CA125) in heart failure (HF) [1].

First, we want to clarify to the authors that longitudinal results presented in our letter entitled "Antigen carbohydrate 125 in heart failure: Not just a surrogate for serosal effusions?" were misinter-

\footnotetext{
* Corresponding author at: Servicio de Cardiología. Hospital Clínico Universitario, Avda. Blasco Ibáñez 17. 46010 Valencia-España. Tel.: +34 652856689; fax: + 34 963862658.

E-mail address: yulnunez@gmail.com (J. Núñez).
}

preted and no methodological issues are present in our calculation [2]. These results (which are also presented in figure 1 - see explanation text at the bottom of the figure), were expressed as median (interquartile range), and not as median (min-max range).

Second, our disagreement with the above authors can be summarized into two aspects: 1 ) the role of CA125 within the very complex and poorly understood pathophysiological cascade that ultimately leads to an elevation of pro-inflammatory markers in acute heart failure syndromes (AHF), and; 2) clinical usefulness of CA125 serum levels in HF.

There is plenty of evidence showing a significant elevation in CA125, proinflammatory markers as well as systemic volume expansion in AHF [3-6]. How these factors are inter-related is still a matter of controversy. Is the mesothelial cells activation by volume expansion/serosal effusions the main mechanism for triggering the production of CA125, or is it the already heightened background inflammation in AHF that triggers the activation of the mesothelial cells, leading ultimately to CA125 elevation? We believe that the amount of evidence available is insufficient to dissect the exact role of CA125 within the (most likely) multifactorial cascade that ultimately leads to an elevation of pro-inflammatory markers in AHF. Therefore, it seems unfair to conclude that CA125 is no more than a simple surrogate for the presence of serosal effusions and mesothelial stimulation. We cautiously have suggested that CA125 may increase in HF patients, not only as a consequence of serosal effusion but, perhaps 